\title{
Stratigraphische Begriffe für das Quartär des Periglazialraums in Deutschland
}

\author{
BRIGITTE URBAN $^{*}$
}

\begin{abstract}
Einleitung: Nur wenige quartäre Vorkommen des Periglazialraums Deutschlands lassen sich paläoökologisch, respektive palynologisch und damit klimatostratigraphisch beschreiben. In diesem Beitrag werden die wichtigsten (bio-)stratigraphischen Begriffe von Warmzeiten und Kaltzeiten des Unter- und Mittelpleistozän, die insbesondere Korrelationen mit den Vereisungsräumen Nord- und Süddeutschlands ermöglichen (z.B. in StePhan \& Menke 1993; LitT et al., in diesem Band), ergänzend beschrieben.

Die chronostratigraphische Gliederung in Unter-, Mittel- und Oberpleistozän erfolgt in Anlehnung an die Stratigraphische Tabelle von Deutschland 2002 (STD 2002), der klimatostratigraphisch begründeten regionalen Gliederung in Kalt- und Warmzeiten für das kontinentale Nordwesteuropa bzw. Deutschland (LiTT et al. 2005), wobei hiernach der traditionellen Pliozän/Pleistozän-Grenzziehung im Bereich des biostratigraphischen Reuver/Prätiglium (ZAGWIJN 1960) gefolgt wird. Die Abschnitte des Prätiglium und des Tiglium-Komplexes fallen nach internationaler Festlegung auf das Profil Vrica (Italien) als Grenzstratotyp in das Gelasium, das noch dem Pliozän zugeordnet wird. Die Grenze Gelasium/Calabrium wurde im Profil Vrica im Top der Olduvai-Magnetozone bei ca. 1,8 Millionen Jahren festgelegt und stellt dieser internationalen Festlegung folgend die Untergrenze des Pleistozäns dar. Die Zweckmäßigkeit dieser Grenzziehung, die vorausgehende einschneidende Klimaereignisse nicht berücksichtigt, wird von zahlreichen Autoren kontrovers diskutiert.

Die Grenze zwischen Unter- und Mittelpleistozän wird im internationalen Konsenz an die paläomagnetisch definierte Brunhes/Matuyama-Grenze bei 780 ka gelegt, das Oberpleistozän beginnt mit dem letzten Interglazial, der Eem-Warmzeit (MIS 5e).
\end{abstract}

\section{Stratigraphical Terms for the Quaternary of the Periglacial Area in Germany}

Keywords: Stratotypes, Quaternary, Periglacial, Germany

Only a few Quaternary deposits of the periglacial area in Germany can be described in paleoecological or palynological - and therefore climatostratigraphical - terms. This paper provides a supplementary description of the most important (bio)stratigraphical terms relating to the warm and cold stages of the Lower and Middle Pleistocene, especially those allowing correlations with the glaciated regions of northern and southern Germany (e.g. in Stephan \& Menke 1993; Litt et al., this volume).

The chronostratigraphical subdivision into Lower, Middle and Upper Pleistocene is based on the Stratigraphical Table of Germany 2002 (STD 2002) and on the climatostratigraphical regional division into cold and warm stages for continental northwestern Europe and Germany (LiTT et al. 2005),

\footnotetext{
*Anschrift der Verfasserin: Prof. Dr. BrigitTe Urban, Universität Lüneburg, Campus Suderburg, Herbert-Meyer-Str. 7, D 29556 Suderburg, Email: b.urban@uni-lueneburg.de
} 
which follows the traditional positioning of the Pliocene/Pleistocene boundary at the transition between the Reuverian and Pretiglian stages (Zagwijn 1960). According to international agreement on the location of the GSSP at Vrica (Italy), the sections of the Pretiglian stage and the Tiglian Complex belong to the Gelasian, which is still assigned to the Pliocene. The Gelasian/Calabrian boundary was set at about 1.8 million years at the Vrica section at the top of the Olduvai magnetozone and therefore represents the internationally agreed base of the Pleistocene. The practical value of this boundary - which does not take prior major climatic events into account - has been a matter of controversy. By international consensus, the boundary between the Lower and Middle Pleistocene has been set at the palaeomagnetically defined Brunhes/Matuyama boundary at $780 \mathrm{ka}$; the Upper Pleistocene begins with the last interglacial, the Eemian stage (MIS 5e).

\section{Unterpleistozän}

\section{Frechen-Kaltzeit Ia, Frechen-Kaltzeit Ib; Fortuna-Oszillation (Fortuna Oszillation)}

\section{Definition, Erstbeschreibung}

Die Bezeichnungen Frechen-Kaltzeit Ia und Ib und Fortuna-Oszillation für eine im Tagebau Frechen, Ville, Niederrheinische Bucht, von KowalczyK (1969) und Boenigk et al. $(1972,1974)$ beschriebene altpleistozäne Schichtenfolge von Tonen und Schotterkörpern wurden von URBAN (1978a, b, 1979) eingeführt.

\section{Typuslokalität}

Typuslokalitäten sind die Tagebaue Frechen und Fortuna der Ville in der südlichen Niederrheinischen Bucht/Nordrhein-Westfalen. Als Typusprofile gelten aus dem Tagebau Fortuna-Garsdorf bei KowALCZYK (1969), BoenigK et al. (1972, 1974) und Urban (1978a, b, 1979) beschriebene Abfolgen mit dem Pollendiagramm auf Beilage Nr. 1 in UrBan (1978a).

\section{Charakteristik, Untergliederung}

BoENIGK et al. (1972) untergliederten diese Schichtglieder der älteren Hauptterrasse (STEEgER 1925) in einen Schotter b1, Ton B1 und den Schotter b2. Intensive als kryogen gedeutete Schichtverwürgungen, petrographische Eigenschaften und Schwermineralspektren weisen den Schotter b1 als pleistozänen Rheinschotter aus (Frechen-Kaltzeit Ia).

Im zwischenlagernden Ton B1 (Fortuna-Oszillation) dominiert Pinus (40-50\%), wärmeliebende Gehölze fehlen fast völlig, Selaginella, Lycopodium, Botrychium und Myricaceae deuten auf boreale Klimabedingungen mit Dauerfrost hin. Der diskordant über dem Tonhorizont B1 liegende Schotter b2 (Frechen-Kaltzeit Ib) weist erstmals ein quartäres Buntschotterspektrum auf.

\section{Vergleichbare Profile, Verbreitung}

Die Fortuna-Oszillation ist in dieser Form bisher nur aus dem Tagebau Fortuna-Garsdorf bekannt.

\section{Datierung}

Nach paläomagnetischen Messungen ist der untere Teil des Tonhorizonts B1 normal, der obere Teil revers magnetisiert (BOENIGK 1978, URBAN 1979) und entspricht damit möglicherweise dem ReunionI-Event.

Die Frechen-Kaltzeit wird von URBAN (1978a) aufgrund vegetationsgeschichtlicher Befunde und ihrer stratigraphischen Position über dem Reuver C (Tonhorizont A11, Serie 11 der Rheinbraun AG) (v.D. Brelie in BoenigK et al. 1974, Urban 1978a) und im Liegenden einer Warmzeit vom Tiglian A Typ (Frechen-Interglazial I, Ville Ton B2, Tagebau Frechen) in das Prätegelen (s.1.) gestellt. Demzufolge wurde die gesamte unterpleistozäne Sedimentfolge (Schotter b1, Ton B1 Schotter b2) der Tagebaue der Ville in das Prätiglium eingestuft (UrBAN, 1978a, b). 
Die Fortuna-Oszillation der Tagebaue der Ville wurde mit Pollenspektren vom Prätegelen-Typ des Tonhorizontes III der Grube Molzmühle in der Region Brüggen (nordwestliche Niederrheinische Bucht) korreliert (URBAN 1978b), die dort dem Typusprofil der Bohrung Meinweg (ZAGwiJn 1960) entsprechen.

\section{Frechen-Interglazial I}

\section{Definition, Erstbeschreibung}

Der Begriff Frechen-Interglazial I wurde erstmals von URBAN (1978b) für den Tonhorizont B2 der unterpleistozänen Schichtenfolge des Tagebaus Frechen verwendet. Er kennzeichnet die auf die prätegelen-zeitliche Sedimentfolge (Schotter b1, Ton B1 Schotter b2: Kowalczyk 1969, Boenigk et al. 1974, BoENigK 1978) der Tagebaue der Ville folgende, ausgeprägte Fagus-führende Warmzeit.

\section{Typlokalität}

Typlokalität ist der auf der Ville, in der südlichen Niederrheinischen Bucht/Nordrhein-Westfalen, gelegene Tagebau Frechen. Als Typusprofil gilt das Pollendiagramm auf Beilage I in Urban (1978b).

\section{Charakteristik, Untergliederung}

Die sandig, schluffig-tonig ausgebildete, ca. $6 \mathrm{~m}$ mächtige warmzeitliche Rinnenfüllung wird von Sedimenten der Frechen-Kaltzeit Ia unterlagert, im Hangenden folgt ein kaltzeitlicher Schotterkörper (Frechen-Kaltzeit II).

Das Pollendiagramm weist Fagus-Pinus-Picea-(Carpinus)-und Tsuga-reiche Pollenzonen auf(UrBAN 1978b), dem von KempF in KowalczyK (1969) nachgewiesenen Vorkommen von Azolla tegeliensis kommt überdies ein biostratigraphischer Leitwert zu.

\section{Vergleichbare Profile, Verbreitung}

Das Frechen-Interglazial I ist aufgrund seiner signifikanten Paläoflora (charakteristisches Vorkommen von Fagus) mit der Pollenzone TA (Tegelen A) der Bohrung Eindhoven II und dem Tegelen A (Belfeld-Ton) (ZAGWIJN 1963) im Raum Brüggen korreliert worden (URBAN 1978a, b). Das Pollendiagramm des Frechen-Interglazial I lässt eine differenziertere und erweiterte Pollenzonierung der Warmzeit gegenüber dem niederländischen Typprofil des Tegelen A zu. Das Frechen-Interglazial I wurde von URBAN (1978 b) mit der Nordende-Warmzeit der Lieth-Serie (MenKe 1975) korreliert.

\section{Datierung}

Der Tonhorizont B2 des Frechen-Interglazials I ist nach Kočı in BoENIGK et al. (1972) revers magnetisiert und könnte demnach im engeren Zeitbereich vor oder nach dem Reunion-II-Event sedimentiert worden sein.

Das Frechen-Interglazials I ist aufgrund seiner biostratigraphischen Charakteristika zweifelsfrei in das Tiglium A der niederländischen stratigraphischen Gliederung einzustufen.

\section{Frechen-Interglazial II (Synonym: Frechen-Kaltzeit II, Frechen-Kaltzeit III)}

\section{Definition, Erstbeschreibung}

Die Bezeichnung Frechen-Interglazial II wurde erstmals von URBAN (1978a) für den Tonhorizont C der unterpleistozänen Schichtenfolge des Tagebaus Frechen eingeführt. Er kennzeichnet den auf den Schotter c, Frechen-Kaltzeit II (Kowalczyk 1969, Urban 1978a, b) der Tagebaue der Ville folgenden, warmzeitlichen Ton C. Das sandig-schluffige, ca. 4 m mächtige warmzeitliche Sediment wird von Grobkieslagen eines Schotterkörpers (Schotter d, Frechen-Kaltzeit III nach URBAN, 1978a) überla- 
gert, den Erosionsdiskordanzen, Driftblöcke und Kryoturbationen als eindeutig kaltzeitliche Bildung ausweisen.

\section{Typlokalität}

Typlokalität ist der auf der Ville in der südlichen Niederrheinischen Bucht/Nordrhein-Westfalen gelegene Tagebau Frechen. Als Typusprofil gelten die Pollenspektren in UrBAN (1978a: 50-54, Beil. 3).

\section{Charakteristik, Untergliederung}

Der Tonhorizont C weist eine rein warmzeitliche Gastropodenfauna überwiegend terrestrischen Milieus (LožEK in BoENIGK et al. 1972) auf und führt häufig Kalkkonkretionen (KowalczyK 1969). Pflanzliche Makroreste (KEMPF in BoENigK et al. 1972) wie auch die eher spärliche Pollenführung weisen auf eine altquartäre warmzeitliche Flora hin (URBAN 1978a).

\section{Vergleichbare Profile, Verbreitung}

Der Tonhorizont C hat auf der Ville eine mehr lokale Verbreitung (BoENIGK 1978).

URBAN (1978a) stellt das Frechen-Interglazial II aufgrund seiner stratigraphischen Position im Hangenden des Frechen-Interglazial I (Tiglium A) und im Liegenden des Frechen-Interglazial III (Tiglium C 5-6, Ton D der Ville-Abfolge) in das Tiglium C1-4 der niederländischen Stratigraphie (ZAGWIJN 1963).

\section{Datierung}

Der Tonhorizont $\mathrm{C}$ des Frechen-Interglazials II ist in seinem unteren Teil revers, im oberen Teil normal magnetisiert (BoENIGK et al. 1972) und könnte demnach an der Wende zum Olduvai-Event sedimentiert worden sein (KLOSTERMANN 1992).

\section{Frechen-Interglazial III}

\section{Definition, Erstbeschreibung}

Die Bezeichnung Frechen-Interglazial III findet sich erstmals bei URBAN (1978a) für den warmzeitlichen Tonhorizont D (KowalczyK 1969) der unterpleistozänen Schichtenfolge des Tagebaus Frechen. Er wird von Grobkieslagen eines Schotterkörpers (Schotter d, Frechen-Kaltzeit III nach UrBAN 1978a) unterlagert, den Erosionsdiskordanzen, Driftblöcke und Kryoturbationen als eindeutig kaltzeitliche Bildung ausweisen.

\section{Typlokalität}

Typlokalität ist der auf der Ville in der südlichen Niederrheinischen Bucht/Nordrhein-Westfalen gelegene Tagebau Frechen. Als Typusprofil gilt die Abfolge mit dem Pollendiagramm auf Beilage Nr. 3 in Urban (1978a).

\section{Charakteristik, Untergliederung}

Der über dem Schotter d auftretende Tonhorizont D mit größerer Verbreitung weist eine warmzeitliche Gastropodenfauna auf und führt neben anderen altpleistozänen makroskopisch nachgewiesenen Florenlementen Azolla filiculoides und Azolla tegeliensis (LožEK \& KempF in Kowalczyк 1969). Den pollenarmen Tonhorizont charakterisiert eine altquartäre warmzeitliche Flora, die im jüngeren Abschnitt des Pollendiagramms überwiegend von Eucommia, Pterocarya, Ostrya und Carpinus charakterisiert wird (URBAN 1978a).

\section{Vergleichbare Profile, Verbreitung}

Der Tonhorizont D tritt auf der Ville als Rinnenfüllung oder in Linsen mit eher lokaler Verbreitung auf. 
Das Frechen-Interglazial III ist von URBAN (1978a) aufgrund seiner stratigraphischen Position im Hangenden des Frechen-InterglaziaI I (Tiglium A) und des Frechen-Interglazial II (Tiglium C1-4) auf der Basis seiner altpleistozänen Makroflorenfloren- und Gastropodenvorkommen und Pollenspektren mit Nachweisen für Azolla tegeliensis mit dem Tiglium C 5-6 der niederländischen Stratigraphie (ZAGWIJN 1963) und den Abschnitten 5-6 der Ellerhoop-Warmzeit (MenKE 1975) korreliert worden.

\section{Datierung}

Der Tonhorizont D des Frechen-Interglazials III (BoENIGK et al. 1972) ist normal magnetisiert und könnte demnach während des Olduvai-Events entstanden sein (Urban 1979, KLOSTERmann 1992).

\section{Van-Eyck-Interglazial}

\section{Definition, Erstbeschreibung}

Die Bezeichnung Van-Eyck-Interglazial wurde erstmals von UrBAN (1978a) für den Tonhorizont V (Nomenklatur nach Boenigk 1970) der Tegelen-Formation in der Belfeld-Region bei Brüggen (nordwestliche Niederrheinische Bucht) benutzt. Der Tonhorizont V wird von URBAN (1978a) aufgrund seiner Paläoflora in der Brüggener Grubenregion nicht insgesamt als Synonym für den von ZAGWIJN (1963) in das Tiglian A gestellten Belfeld-Ton beschrieben.

\section{Typlokalität}

Typlokalitäten der Van-Eyck-Interglazial Warmzeit sind die Gruben Peter van Eyck (Nr. 104) und benachbarte Tongruben (Nr. 102, 103, 105 Nummerierungen nach BoENIGK 1970) bei Brüggen in der nordwestlichen Niederrheinischen Bucht/Nordrhein-Westfalen. Als Typusprofile gelten die Pollendiagramme auf Beilage Nr. 5, 6 und 9 in UrBAn (1978a).

\section{Charakteristik, Untergliederung}

Maßgebliche Florenelemente sind Pterocarya, Eucommia, Decodon, Phellodendron, Carya, Parthenocissus bei völliger Abwesenheit von Fagus. Wiederholt wurde eine starke Abkühlung im oberen Teil des Tonhorizontes V beobachtet. Azolla tegeliensis und Azolla filiculoides treten vereinzelt im Ton V auf (KempF in Boenigk, 1970), der von Urban (1978a) in das Tiglium C5-6 gestellt wurde. Aufgrund schwermineralogischer Untersuchungsergebnisse wurde der Ton V von BoenigK (1970) als Waal-zeitlich oder jünger angesehen.

\section{Vergleichbare Profile, Verbreitung}

Der Tonhorizont V hat im Raum Brüggen als Altarmablagerung des Paläorheins weite Verbreitung. Seine Paläoflora und biostratigraphische Abgrenzung zum liegenden Tonhorizont III der BelfeldRegion (Reuverium B, Zagwisn 1974b; Reuverium B/C, Pretiglium, Urban 1978a, ZagwiJn 1998) und zum Belfeld-Ton (Tiglian A, ZAGWIJN 1963) benachbarter Vorkommen lassen eine zweifelsfreie Einstufung in das Tiglium C5-6 des benachbarten Tegelen-Tons (ZAGwiJn 1963; Cycle III: ZAGwiJN 1998) der Typusregion zu. Das Van-Eyck-Interglazial wurde von URBAN (1978a) mit dem Ton D der Ville Abfolge und der Ellerhoop-Warmzeit 5-6 (MenKe 1975) verknüpft.

\section{Datierung}

Der Ton V ist nach Kočı in Boenigk et al. (1972) unten revers und oben normal magnetisiert, nach neueren Messungen (KočI \& SiBrava 1976, URBAN 1979) ist er auch in seinem tieferen Teil normal magnetisiert. Eine Einstufung in das Olduvai-Event ist daher wie für den Tonhorizont D der Ville naheliegend (UrBAN 1979, vgl. auch ZAGWIJN 1998). 


\section{Mittelpleistozän}

\section{Frimmersdorf-Interglazial \\ (Synonym „Interglazial von Frimmersdorf“; „Frimmersdorfer-Interglazial“)}

\section{Definition, Erstbeschreibung}

Der Begriff „Interglazial von Frimmersdorf“ wurde für tonig-humose Ablagerungen der Oberen Mittelterrasse I im Tagebau Frimmersdorf-West von v.D. Brelie et al. (1959) eingeführt. Schirmer (1974) benutzte die Bezeichnung „Frimmersdorfer-Interglazial“ für Abfolgen im gleichen Aufschluss.

\section{Typlokalität}

Typlokalität für das „Interglazial von Frimmersdorf“ synonym dem „Frimmersdorfer-Interglazial“ und dem „Frimmersdorf-Interglazial“ sind die Tagebaue Frimmersdorf-West und Fortuna-Garsdorf der südlichen Niederrheinischen Bucht/Nordrhein-Westfalen. Als Typusprofil gelten das Pollenprofil in V.D. BRELIE et al. (1959: 182-183) und für den Tagebau Fortuna-Garsdorf in URBAN (1980a: 226).

\section{Charakteristik, Untergliederung}

Im dreigeteilten Pollendiagramm dominieren Pinus und Salix an der Basis, Alnus mit Eichenmischwaldanteilen $(<5 \%)$ im mittleren und Pinus mit z.T. $<80 \%$ im obersten Abschnitt. Das Auftreten von Juglans, Pterocarya und eines Keteleeria-ähnlichen Pollentyps werden als autochthon beschrieben (v.D. BRelie et al. 1959). Holz- und andere makroskopische Pflanzenreste erweitern das Florenspektrum um Vertreter der Buxaceae (Buxus sempervirens), Aquifoliaceae, Staphyleaceae, Aceraceae und Caprifoliaceae, auch das Vorkommen von Azolla filiculoides und Vitis sylvestris ist belegt. Die übrigen palynologischen Befunde deuten auf kühlere Klimabedingungen während des Ablagerungszeitraumes hin. Guterhaltene Insektenreste stammen von kälteliebenden Spezies. Die durchgehend sehr hohen Pollengehalte miozäner Florenelemente deuten auf Umlagerung hin. Das Vorkommen wurde aufgrund der Lagerungsverhältnisse seinerzeit als Mindel-Riß-Interglazial (v.D. BRELIE et al. 1959) angesprochen.

\section{Vergleichbare Profile, Verbreitung}

In dieser Form ist das Frimmersdorf-Interglazial (Interglazial von Frimmersdorf, FrimmersdorferInterglazial) nur aus den Tagebauen der Ville bekannt. Im Tagebau Fortuna-Garsdorf wurde eine von BRUNNACKER et al. (1978) über Kiesen der Mittelterrasse II a liegende, analoge tonig-schluffige Ablagerung „Frimmersdorfer-Interglazial“ beschrieben, die von URBAN (1980a) palynologisch untersucht und als Frimmersdorf-Interglazial bezeichnet wurde. URBAN (1980a) beschreibt einen basalen Diagrammabschnitt, der schon weitgehend von einem reichen Kräuterspektrum beherrscht wird, das im jüngsten Profilabschnitt verarmt und in eine Artemisia-Steppe übergeht. Im Einklang mit der Molluskenfauna (LožEK 1964) mit Bythynia tentaculata steht daher die Annahme (UrBAN 1980a), dass der Ablagerungsbeginn gegen Ende eines Interglazials oder Interstadials lag und zum Hochglazial im Hangenden überleitet. Eine Einstufung in einen späten Abschnitt des Cromer-Komplexes wird von BRUNNACKER et al. (1978) und URBAN (1980a) vorgeschlagen.

\section{Datierung}

Nach paläomagnetischen Messungen ist der schluffige Ton des Frimmersdorf-Interglazials im Tagebau Fortuna-Garsdorf normal magnetisiert und gehört damit in die Brunhes-Chron (Kočı, in BRUNNACKER et al. 1978). Mit numerischen Methoden der Altersbestimmung ermittelte absolute Daten liegen nicht vor, so dass sich nur eine vorläufige Zuordnung zur MIS 17 ergibt (KLOSTERMANN 1992).

Eine Einstufung in den späten Cromer-Komplex ist von BRUNNACKER et al. (1978) und URBAN (1980a) und in ältere Abschnitten von Klostermann (1992) vorgenommen worden. BoenigK (1995) stellt das Terrassenniveau des Frimmersdorf-Interglazials in das jüngere Niveau der Mittelterrasse III. Von BoENIGK \& FRECHEN (1998) wird darauf aufbauend eine Verknüpfung mit der Deckschichtenstratigraphie 
der Pleistozänabfolge von Kärlich (Mittelrhein) vorgenommen und das Frimmersdorf-Interglazial mit dem „Kärlicher Interglazial I“ korreliert, tentativ in das Holstein-Interglazial, also MIS 9 gestellt.

\section{Krefeld-Interglazial}

\section{Definition, Erstbeschreibung}

Der Begriff Krefeld-Interglazial wurde von URBAN (1980b) für warmzeitliche schluffig-tonige Altwassersedimente gestauchter Vorkommen, den sogenannten „Krefelder Schichten“ (BERTSCH \& STEEGER 1927) aus dem Bereich der Unteren Mittelterrasse bei Krefeld, eingeführt.

\section{Typlokalität}

Typlokalität für das Krefeld-Interglazial sind die gestauchten Krefelder-Schichten im Bereich der südlichen Stauchmoräne des Xantener Lobus, der Inselberge (z.B. Eyller Berg) und des Schaphuysener Höhenzuges. Als Typusprofil für das Krefeld-Interglazial gelten insbesondere die Profile Römerhof I und II in URBAN (1980b:76-77).

\section{Charakteristik, Untergliederung}

Die Pollenspektren des Krefeld-Interglazial zeigen bei relativer Gleichförmigkeit Koniferen-Dominanz (Pinus, Abies), daneben treten Auenwaldelemente (Pterocarya, Alnus, Vitis), Pollen von Fagus, Buxus und Taxus ebenso wie Massulae von Azolla filiculoides in den untersuchten Profilen auf. Auffallend sind besonders hohe Anteile von Corylus, die in den Profilen der südlichen Stauchmoräne des Xantener Lobus (Römerhof) im Gegensatz zu denen des Eyller Berges und des Schaphuysener Höhenzuges (Stenden) zu stehen scheinen. Der malakozoologische Befund (PuissÉGUR in Urban 1980b) mit Valvata piscinalis und Bythynia tentaculata deutet auf interglaziale Ablagerungsbedingungen entlang eines durch wechselnde Strömungsgeschwindigkeiten charakterisierten Flusslaufes hin.

\section{Vergleichbare Profile, Verbreitung}

Am Niederrhein sind in der weiteren Umgebung von Krefeld im Bereich der Verbreitung der Krefelder Mittelterrasse und der rheinwärts angrenzenden saalezeitlichen Stauchendmoränen zahlreiche Ton- und Schluffvorkommen verbreitet, die als „Kempener-Schichten“ (BERTSCH et al. 1931) ungestörte Schotter unterteilen und eingeschuppt in die Stauchmoränen als Krefelder-Schichten (BERTSCH \& Steeger 1927) bekannt sind. Pollenanalytisch wurden beide Schichten von Karrenberg \& Rein (1951), V.D. BRELIE \& ReIN $(1952,1956)$ und später auch von KempF (1966) umfangreich paläoökologisch untersucht (Profil Tönisberg), als Kempen-Krefelder Schichten zusammengefasst und in das Holstein-Interglazial gestellt. Demgegenüber steht die Auffassung von Steusloff in Bertsch et al. (1931), dass die Molluskenfauna der Kempener-Schichten sich grundsätzlich von den Vergesellschaftungen der Krefelder-Schichten unterscheide und die Ansicht von KAISER \& SCHÜTRUMPF (1960), die Krefelder Mittelterrasse und damit die Kempener-Schichten seien jünger als der saalezeitliche Eisvorstoß.

Von URBAN (1980a, b) werden die interglazialen Tonvorkommen der „Krefelder-Schichten“ in den Stauchwällen und Inselbergen als gleich alte Bildungen beschrieben und in das Holstein-Interglazial (s.1.) gestellt.

Klostermann (1992) differenziert die interglazialen Tone und Schluffe in einen Holstein I Typ (Holstein s.1.) für Schichten mit einem Pollenspektrum des Krefeld-Interglazials und in einen Holstein II Typ (Holstein s. str.), dem insbesondere das bei KempF (1966) beschriebene Profil des Schachtes Tönisberg zugeordnet wird. BoENIGK \& FRECHEN (1998) fassen die Kempen-Krefelder Schichten erneut zusammen und stellen sie in die Saale s.1.. Im Krefeld-Interglazial sehen diese Autoren das Korrelativ zum Kärlich Interglazial s.str. nach URBAN (1978c, 1983) und den analogen Abfolgen von BitTMANN (1991), das sie als Kärlicher Interglazial II bezeichnen. 


\section{Datierung}

Mit numerischen Methoden der Altersbestimmung ermittelte absolute Daten liegen nicht vor.

\section{Kärlicher Interglazial}

\section{Definition, Erstbeschreibung}

Der Begriff Kärlicher Interglazial wurde ursprünglich von BRUNNACKER et al. $(1969,1975)$ und BRUNNACKER (1971) für den oberen Teil des Abschnittes H der Deckschichtenabfolge der Tongrube Kärlich am Mittelrhein eingeführt.

\section{Typlokalität}

Als Typlokalität für das Kärlicher Interglazial gilt die Tongrube Kärlich am Mittelrhein/RheinlandPfalz. Der Begriff Kärlicher Interglazial ist auf die warmklimatische Abfolge des oberen Abschnittes $\mathrm{H}$ der Deckschichtenabfolge der Tongrube Kärlich unterhalb des Brockentuff (URBAN 1978c) festgelegt.

\section{Charakteristik, Untergliederung}

Abschnitt H schließt im oberen Teil mit einer Braunerde (Frechen \& RosenAuer 1959, MücKenhauSEN 1959), nach BRUNNACKER (1971) mit einer Parabraunerde ab, die interglaziale Mollusken führt, überlagert von Ablagerungen des Kärlich-Brockentuffes und Sedimenten einer in ihn eingetieften Depression (BRunNACKer, in Bosinski et al. 1980) ab. SchiRmer $(1970,1990)$ stellt den warmklimatischen Charakter der Bildungen an der Basis des Kärlich-Brockentuff zudem aufgrund von Florenresten fest.

VAn Kolfschoten \& TuRner (1996) beschreiben eine Kleinsäugerfauna mit Talpa sp., Scriuridae gen. sp., Microtus arvalis/Microtus agrestis, Microtus sp. und Apodemus sp. aus einem analogen Horizont unterhalb des Brockentuffs. Nach BoenigK \& FreChen (1998) wird die Obergrenze des Abschnitts $\mathrm{H}$ des Deckschichtenprofils in Kärlich durch den Kärlich-Brockentuff definiert, der bereits an den Anfang von Abschnitt J gestellt wird.

\section{Vergleichbare Profile, Verbreitung}

Die Straten des oberen Abschnittes H, dem Ende der Bildung von Schicht H der Tongrube Kärlich (BRUNNACKER 1971) bleiben auf die Fundlokalität beschränkt.

(Siehe hierzu Kapitel Kärlich-Interglazial („Kärlich Interglazial“)).

\section{Datierung}

(Siehe hierzu Kapitel Kärlich-Interglazial („Kärlich Interglazial“))).

\section{Kärlich-Interglazial \\ (Synonym „Kärlich Interglazial““, „Kärlich Interglacial“)}

\section{Definition, Erstbeschreibung}

Der Begriff Kärlich-Interglazial („Kärlich Interglazial“) wurde von URBAN (in BosINSKI et al. 1980) und URBAN (1983) für warmzeitliche, limnisch-telmatische Sedimente einer Reliefdepression im Kärlich-Brockentuff der Tongrube Kärlich am Mittelrhein eingeführt.

\section{Typlokalität}

Als Typlokalität für das Kärlich-Interglazial gilt die Tongrube Kärlich am Mittelrhein/RheinlandPfalz mit dem bei Urban (1983: 85-88) beschriebenen Pollendiagramm und seiner Zonierung. Der Begriff „Kärlich-Interglazial“ („Kärlich Interglazial“, Kärlich Interglacial) ist auf die pollenanalytisch beschriebene Sedimentabfolge einer Depression über dem Brockentuff (URBAN 1978c) festgelegt. 


\section{Charakteristik, Untergliederung}

Urban (1983: 85, 88) beschreibt ein in fünf Pollenzonen (K 1-5) untergliedertes Diagramm, das in der Eichenmischwaldphase eines Interglazials einsetzt, der ein ausgeprägter Corylus Gipfel, eine Hainbuchen-Tannen-, Hainbuchen-Birken- und Pinus-Picea- Phase folgen. Die in einer prähistorischen Fundschicht (Bosinski et al., 1980) aus der Hainbuchen-Birkenphase stammenden Holzkohlenreste sind den Taxa Abies alba, Celtis australis und Acer spec. (Sсносн in Urban 1983) zuzuordnen, Pollen von Pterocarya und Fagus sowie Massulae von Azolla filiculoides wurde ebenfalls belegt (URBAN 1983).

Umfangreiche vegetationsgeschichtliche Untersuchungen der Sedimentabfolge „Kärlich-Seeufer“, einer Depression über dem Brockentuff, durch BitTMAnN $(1990,1991)$ bestätigen detailliert den durch Urban (1978c, 1983) gemachten Befund. Die archäologischen Fundschichten des KärlichInterglazial werden als Lokalität „Kärlich-Seeufer“ bei KRÖGER et al. (1991) und GAUDZINSKI et al. (1996) zusammenfassend beschrieben. BitTMANN (1990: 44-52) beschreibt acht Pollenzonen mit interglazialem Charakter, gefolgt von einer waldfreien Phase, an die sich eine Kiefern-Fichten-Eichen Zeit (Z11 Interstadial) anschließt, auf die ein erneuter Klimarückschlag folgt. Die Sedimentation der interglazialen Schichten setzt in der Eichenmischwaldzeit ein. Unter den Großsäugerresten befinden sich Elephas antiquus, Equus sp., Cervus elaphus, Sus scrofa und Bos sp./Bison sp. (van Kolfschoten \& TURNER 1996).

\section{Vergleichbare Profile, Verbreitung}

In der Tongrube Kärlich gilt die bei Bittmann (1990, 1991) pollenanalytisch beschriebene Abfolge der Lokalität „Kärlich-Seeufer“ als Äquivalent des Kärlich-Interglazial („Kärlich Interglazial“‘) s.str. nach URBAN (1983).

Die Bildungen an der Basis des Kärlich-Brockentuffs als auch der Brockentuff selber und die in ihn eingetiefte Depression wurden von BRUNNACKER et al. (1969) und BRUNNACKER, in Bosinski et al. (1980) in den oberen Abschnitt H der Deckschichtenabfolge von Kärlich einbezogen, und die Eruption des Brockentuffes in den mittleren Bereich einer Warmzeit gestellt (BosinsKI et al. 1980, URBAN 1978c, Bittmann 1990). Für diese Gesamtabfolge wird teilweise die Bezeichnung Kärlicher Inter-

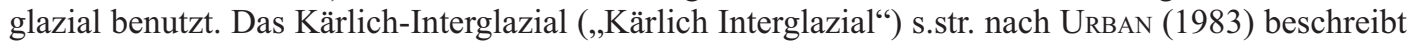
jedoch nur die limnisch-telmatischen Sedimente der Depression oberhalb des Kärlich-Brockentuffes. Aufgrund der nicht immer eindeutigen terminologischen Trennung in Kärlicher Interglazial und Kärlich-Interglazial („Kärlich Interglazial“), der unterschiedliche genetische Interpretationen für den Abschnitt H (nach BRUNNACKER in BosinsKi et al. 1980) zugrunde liegen und aufbauend auf jüngere Forschungsergebnisse, schlagen BoenigK (1995) und BoENIGK \& FreCHEN (1998) vor, in ein Kärlicher Interglazial I (warmzeitliche Bildung im Liegenden des Brockentuffes, Typsedimente des Kärlicher Interglazial, BRUNNACKER et al. 1969) und Kärlicher Interglazial II Kärlich-Interglazial („Kärlich Interglazial“") s.str., URBAN 1983) zu trennen. Zwischen den beiden warmklimatischen Phasen liegen nach BOENIGK \& FRECHEN (1998) eine Erosionsphase, zwei pedogen überprägte Fließerde Sedimentationszyklen mit ökologisch differenzierter, warmklimatischer Molluskenfauna und die Eruption und der Versturz des Kärlich-Brockentuffs mit Bildung der Depression des Kärlich-Interglazial („,Kärlich Interglazial"“).

Nach Boenigk \& Frechen (1998) wird die Obergrenze des Abschnitts H des Deckschichtenprofils in Kärlich durch den Kärlich-Brockentuff definiert, der bereits an den Anfang von Abschnitt J gestellt wird. URBAN (1983) stellt das Kärlich-Interglazial („Kärlich Interglazial“) aufgrund seiner intermediären Paläoflora, die Elemente des Eem- als auch des Holstein-Interglazials aufweist, in den Saale-Komplex und diskutiert es als Korrelativ zu Wacken (Menke 1968a), Dömnitz (ERD 1973) und Hoogeveen (ZaGWIJN 1973).

Bittmann (1990, 1991) und BitTMAnN \& Müller (1996) stellen das Kärlich-Interglazial („Kärlich Interglazial") in einen jüngeren Abschnitt des Cromer Komplexes und korrelieren es mit dem Interglazial von Bilshausen/Niedersachsen (MüLLER 1965).

In der Niederrheinischen Bucht weisen die Profile vom Typ „Römerhof“ des Krefeld-Interglazials 
(URBAN 1980a) vegetationsgeschichtlich Parallelen zum Kärlich-Interglazial („Kärlich Interglazial“) auf. BOENIGK \& FRECHEN (1998) korrelieren die Kempen-Krefelder Schichten in ihrer Gesamtheit mit dem Kärlich-Interglazial („Kärlich Interglazial“) s.str. (=Kärlicher Interglazial II) und stellen es in den Saale-Komplex.

\section{Datierung}

Methodisch wenig verlässliche ${ }^{230} \mathrm{Th} / \mathrm{U}$-Datierungen von im Brockentuff enthaltenen Kalkonkretionen (HENNING, in Bosinski et al. 1980) lieferten ein Mindestalter von $150 \mathrm{ka}$, Thermolumineszenz-Datierungen (TL) an Löss im Hangenden und Liegenden (ZöLLER et al. 1988) des Kärlich-Brockentuffs ebenfalls Minimalwerte von $152 \pm 15,222 \pm 25$ und $232 \pm 28$ ka. VAN DEN BoOgAARD et al. (1989) führten ${ }^{40} \mathrm{Ar} /{ }^{39} \mathrm{Ar}$-Laserdatierungen an Phlogopit-Einzelkristallen des Brockentuffs durch und ermittelten ein Eruptionsalter von $396 \pm 20 \mathrm{ka}$. Einer neueren Datierung zufolge stellt BoogARD (in BoENIGK \& FreChen 1998) die Eruption des Brockentuffs in den Anfang von MIS 10.

\section{Mauerer Waldzeit \\ (Synonym Warmzeit von Mauer (Grafenrain))}

\section{Definition, Erstbeschreibung}

Der Begriff Mauerer Waldzeit ist für die Mauerer Neckarsande (untere Mauerer Sande) mit nicht eindeutig rekonstruierbarer Priorität geprägt worden, die den Unterkiefer des Homo heidelbergensis von Mauer (gefunden von DANiEL HARTMANN, 21.10.1907, SCHOETENSACK 1908) und zahlreiche für warmklimatische Verhältnisse sprechenden Großsäugerfaunenreste führen (zusammengefasst in BEINHAUER \& WAGNER 1992, 1997).

\section{Typlokalität}

Typlokalität für die Mauerer Waldzeit ist die Sandgrube Grafenrain in Mauer bei Heidelberg, Typus Stratum sind die Unteren Mauerer Sande mit der Fundschicht des Homo heidelbergensis.

\section{Charakteristik, Untergliederung}

Die Mauerer Waldzeit ist anhand der Mikromammalia- und Großsäugerfaunenfunde vor allem in der ersten Hälfte des letzten Jahrhunderts (Schoetensack 1908, Freudenberg 1911, 1914, Soergel 1914, Heller 1934) beschrieben worden. Soergel (1928) beschreibt das geologische Alter des Homo heidelbergensis mit 450.000 Jahren und nimmt zur geologischen Entwicklung der Neckarschlinge von Mauer Stellung (Soergel 1933). Später gliedert GraUl (1977, zusammengefasst in Löscher 1997), die fluviatilen Ablagerungen des Neckar und der Elsenz in der Mauerer Schleife. Durch v. Koenigswald $(1992,1997)$ und v. Koenigswald \& HeinRich (1999) sind die Faunen der Mauerer Sande in jüngerer Zeit umfangreich biostratigraphisch bearbeitet worden. Aus v. KoENIGSwald \& HeInRICH (1999: 84) wird die nachfolgende Faunenliste wiedergegeben:

\section{Säugetierfauna}

Insectivora

Talpa minor

Talpa europaea

Primates

Homo heidelbergensis

Rodentia

Apedumus spec.
Microtus arvalis-agrestis

Arvicula cantianus

Pliomys episcopalis

Castor fiber

Trogonterium cuvieri

Carnivora

Canis lupus mosbachensis

Ursus thibetanus

Ursus deningeri 
Pliocrocuta perrieri

Panthera pardus sickenbergi

Panthera leo fossilis

Felis (Lynx) issidorensis

Felis cf. sylvestris

Homotherium spec.

Proboscidea

Elephas antiqus

Perissodactyla

Equus mosbachensis
Stephanorhinus hundsheimensis

Stephanorhinus kirchbergensis

Artiodactyla

Sus scrofa

Hippopotamus amphibius antiquus

Alces latifrons

Cervus elaphus

Capreolus priscus

Bison schoetensacki

Von Koenigswald \& Heinrich (1999) heben die Neueinwanderung von Arvicula, Elephas antiquus, Hippopotamus amphibius und Homo heidelbergensis in dieser Warmzeit besonders hervor. Aufgrund des für jüngere mittelpleistozäne Interglaziale typischen und in Mauer nur sehr untergeordneten Vorkommens von Stephanorhinus kirchbergensis stellen von Koenigswald \& Heinrich (1999) die Faunen von Mauer in das mittlere Mittelpleistozän und stufen diese älter als die Faunen von Mosbach 2 ein.

Mit Hilfe zweier Forschungsbohrungen (Grafenrain I und II) wurden die pleistozänen Liegendschichten der unteren Mauerer Sande bis auf die Basislagen des Unteren Muschelkalk erschlossen. Im Liegenden der bis $5 \mathrm{~m}$ Tiefe anstehenden Unteren Mauerer Sande der Bohrung I (Gesamtmächtigkeit $22 \mathrm{~m}$ ) folgen bis in $16 \mathrm{~m}$ Tiefe Wechsellagen von schluffigem Ton, Mudden und humosen Sanden, die von Holz und Torfschmitzen durchsetzt sind, die als vermeintliche Altwasserabsätze interpretiert werden (Zöller 1997, Urban 1992, 1997). Die pollenführenden Schichten in 6,9 bis 10,0 m Tiefe konnten von URBAN $(1992,1996,1997)$ in eine Wechselfolge von zwei Interstadial-Kaltzeit-Sequenzen, die der „Mauerer Waldzeit“, insgesamt als „Mauerer Warmzeit“" (URBAN 1992, 1997) bezeichnet, vorausgegangen sind, untergliedert werden. Zwischen 10,00 m und 9,00 m ist eine Kiefern-FichtenErlen-Zeit, die interstadialen Florencharakter hat, erfasst. Darauf folgt zwischen 8,90 m und 7,20 m eine intensive Klimaverschlechterung, belegt durch gräser-kräuerreiche Vegetationsspektren, die von einem zweiten nadelholzreichen Interstadial mit einer Wiederausbreitung von Kiefer und Birke, begleitet von einer Wacholder-Weidenphase, eingeleitet werden.

Diese vegetationsgeschichtlichen Ergebnisse werden von URBAN $(1992,1997)$ zusammengefasst folgendermaßen interpretiert: der Mauerer Warmzeit (Fundschicht) gehen zwei kühle Waldzeiten mit Taiga-artigen Florenelementen, unterbrochen von einer waldfreien Kaltzeit, unmittelbar voraus. MüLLER (1986) beschreibt zahlreiche, derartige kühle Thermomere aus jüngeren Cromer-zeitlichen Ablagerungen der Bohrung Gorleben. URBAN (1997) stuft die Sedimente im Bereich der Mauerer Sande daher in die zweite Hälfte des Cromer-Komplexes ein.

\section{Vergleichbare Profile, Verbreitung}

Die Verbreitung der Mauerer Sande ist auf die unmittelbare Umgebung der Neckarschlinge von Mauer begrenzt.

\section{Datierung}

Die Fundschicht des Unterkiefers des Homo heidelbergensis (Mauerer Waldzeit), insgesamt als Mauerer Warmzeit (URBAN 1992, 1997) bezeichnet, wurde mit verschiedenen naturwissenschaftlichen Ansätzen unterschiedlich eingestuft (Horn et al. 1997, von Koenigswald 1997, Urban 1997, Wagner et al. 1997, ZöLLER 1997). Aufgrund der normalen paläomagnetischen Magnetisierung, der Faunenzugehörigkeit, der Palynologie sowie der Deckschichtenpaläopedologie und weiterer physikalischer Bestimmungsmethoden, ergibt sich das Bild der Tabelle 1. 
URBAN (1997) und ZÖLLER et al. (1997) konstatieren aufgrund einer vegetationsgeschichtlichen Parallelisierung mit Abfolgen der niederländischen und norddeutschen Pleistozän-Abfolgen, dass - sofern kein wesentlicher Hiatus zwischen der jüngeren Waldzeit von Grafenrain (2. kühle Waldzeit) und den Maurer Sanden (interglaziale Fundschicht) vorhanden ist - die Mauerer Warmzeit in die Wende zwischen MIS 16/15 bzw. MIS 14/13 fällt.

Tab. 1: Mögliche Zuordnung der Mauerer Waldzeit zu Marinen Sauerstoffisotopenstufen (MIS)

Tab. 1: Possible correlations of the Mauer-Waldzeit with MIS (marine isotope stages)

\begin{tabular}{|c|c|c|c|}
\hline $\begin{array}{l}\text { Stratum } \\
\text { Untere } \\
\text { Mauerer } \\
\text { Sande }\end{array}$ & $\begin{array}{l}\text { Vegetationsgeschichtlicher } \\
\text { Abschnitt, klimatische Wertigkeit } \\
\text { (URBAN 1997, ZÖLLER 1997) }\end{array}$ & $\begin{array}{l}\text { Tentative Zuordnung } \\
\text { zur MIS-Zeitskala } \\
\text { Alternative a } \\
\text { (URBAN 1997, ZÖLLER } \\
\text { 1997) }\end{array}$ & $\begin{array}{l}\text { Tentative Zuordnung } \\
\text { zur MIS-Zeitskala } \\
\text { Alternative b } \\
\text { (URBAN 1997, } \\
\text { ZÖLLER 1997) }\end{array}$ \\
\hline & Mauerer Waldzeit & MIS 13 & MIS 15 \\
\hline & $\begin{array}{l}\text { ? kaltes, nicht extremes } \\
\text { Wald(steppen)klima }\end{array}$ & MIS 14 & MIS 16 \\
\hline & 1. Kühle Waldzeit & MIS 15 & $?$ \\
\hline & Waldfreie Kaltzeit & MIS 16 & $?$ \\
\hline & $\begin{array}{l}\text { Kaltklima } \\
\text { 1. Kühle Waldzeit }\end{array}$ & $\begin{array}{l}\text { MIS } 18 \\
\text { MIS } 17\end{array}$ & $\begin{array}{l}\max . \text { MIS } 18 \\
?\end{array}$ \\
\hline
\end{tabular}

